\title{
Psychogenic nonepileptic seizures as a manifestation of psychological distress associated with undiagnosed autism spectrum disorder
}

This article was published in the following Dove Press journal:

Neuropsychiatric Disease and Treatment

18 January 2016

Number of times this article has been viewed

\author{
Dai Miyawaki' \\ Yoshihiro Iwakura' \\ Toshiyuki Seto \\ Hiroto Kusaka' \\ Ayako Goto' \\ Yu Okada' \\ Nobuyoshi Asada' \\ Erika Yanagihara' \\ Koki Inoue' \\ 'Department of Neuropsychiatry, \\ ${ }^{2}$ Department of Pediatrics, Osaka \\ City University Graduate School of \\ Medicine, Asahi-machi, Abeno-ku, \\ Osaka, Japan
}

\begin{abstract}
Psychogenic nonepileptic seizures (PNES) are observable changes in behavior or consciousness that are similar to epileptic seizures but are not associated with electrophysiologic changes. PNES occur in children with underlying psychological distress and are especially frequent in those with epilepsy. Because PNES are heterogeneous, comprehensive treatment tailored to each patient is required to reduce psychosocial stress. Currently, reports regarding children with PNES concomitant with autism spectrum disorder (ASD) do not exist, and effective treatment strategies for these children are lacking. In this case report, we describe a 10-yearold Japanese girl with undiagnosed ASD who developed PNES while undergoing treatment for benign childhood epilepsy with centrotemporal spikes. She exhibited hypersensitivity to sound and interpersonal conflicts caused by social communication deficits. The PNES symptoms improved shortly after our intervention, which was designed to reduce her distress caused by auditory hypersensitivity and impaired social communication, both characteristics of ASD. To the best of our knowledge, this is the first report describing PNES in a child with ASD. Our findings suggest that PNES can result from psychological distress in children with undiagnosed ASD and highlight the importance of examining ASD traits in patients with PNES.
\end{abstract}

Keywords: pseudoseizures, autism spectrum disorders, undiagnosed, children

\section{Introduction}

Psychogenic nonepileptic seizures (PNES) are observable changes in behaviors or consciousness that are similar to epileptic seizures but are not associated with electrophysiologic changes. ${ }^{1-3}$ They are considered a conversion disorder that reflects underlying psychological distress and are a major neuropsychiatric problem associated with epilepsy. ${ }^{4}$ Misdiagnosing PNES harms the patients and the health care system because it results in improper treatment of presumed epilepsy and a higher risk of iatrogenic injury and morbidity. ${ }^{5}$ While rare in children without epilepsy, children with epilepsy often suffer from PNES. ${ }^{6}$ Because PNES are heterogeneous in nature, multidisciplinary strategies aimed at reducing precipitating psychosocial stressors have been emphasized, ${ }^{1,3,7-9}$ despite a lack of controlled studies in pediatric populations.

Autism spectrum disorder (ASD) is a group of neurodevelopmental disorders characterized by social and communication impairments, repetitive behaviors, and problems with sensory reactivity. ${ }^{9,10}$ Epilepsy is known to often occur in children with ASD. ${ }^{11}$ Because children with epilepsy develop PNES more frequently than those without epilepsy, ${ }^{1}$ children with ASD may suffer from PNES more often than those without ASD. However, the published reports of PNES in children with ASD do not yet exist, and effective treatment strategies for PNES in these children are lacking.
Department of Neuropsychiatry, Osaka City University Graduate School of Medicine, Asahi-machi, Abeno-ku, Osaka 545-8585, Japan

Tel $+8 \mid 66645382$ I

Fax +81666360439

Email miyawaki@med.osaka-cu.ac.jp 
In this report, we describe a case of PNES in a 10-year-old Japanese girl with epilepsy and undiagnosed high-functioning ASD. Our treatment strategy, targeting the ASD characteristics and the overlooked distress associated with auditory hypersensitivity and social communication impairment, resulted in an improvement in her PNES. The Ethics Committee of Osaka City University does not require approvals for a case report. Written informed consent was obtained from the patient and her parents for publication of this case report.

\section{Case presentation}

The subject is a 10-year-old Japanese girl who had her first seizure while asleep at the age of 7. She was subsequently diagnosed with benign childhood epilepsy with centrotemporal spikes by a pediatric neurologist. Electroencephalogram revealed abundant focal spike discharges from the right centrotemporal region during sleep (especially during sleep-stages SI and SII). Her seizures occurred within 1 hour of going to sleep and were accompanied with twitching of the face and arm on the left side. Because of progressing secondarily generalized tonic-clonic seizures, she was prescribed valproate at the age of 7 after having carbamazepine-induced skin reactions. The secondarily generalized seizures were controlled by the medication, and for some time, only focal seizures occurred a few times a year. However, at the age of 10, she developed atonic-like seizures of her legs and tonic-like seizures (accompanied with screams of "ahhh, ahhh") several times a day during wakefulness. As a result, she was immediately admitted to the Pediatric Medical Center of Osaka City University Hospital. She presented with various symptoms more than ten times a day, including thrashing different parts of her limbs in each episode, unresponsiveness, impaired consciousness, and atonic seizures in which she leaned on her mother and uttered "ahhh". Her eyes were closed during these episodes. Although a pediatric neurologist administered her levetiracetam in addition to valproate, her seizures did not improve. No illness underlying her convulsions was identified by several medical examinations, including a brain magnetic resonance imaging, a nerve-conduction study, and a blood test. Compared with the resting (interictal) electron encephalography (EEG), video-EEG revealed no epileptic discharges, such as spikes, polyspikes, or spike and waves during the ictal periods. No continuous fast wave or any change in the basic rhythmic activity was observed during the ictal period, except for motion artifacts. She was ultimately diagnosed with definite PNES and referred to our child psychiatry department.

We performed a comprehensive interview of the subject and her mother to evaluate her developmental history using the Pervasive Developmental Disorders Autism Society Japan Rating Scale, ${ }^{12}$ a standardized semistructured interview useful for children and adults, and the Japanese version of the Schedule for Affective Disorders and Schizophrenia for School-Age Children - Present and Lifetime Version. ${ }^{13}$ The subject has been exceeding the cutoff score for ASD on the former test since early childhood and to the present. The latter test shows that she has had no comorbid psychiatric disorders. Additionally, the Children's Global Assessment Scale revealed that while her global functioning was categorized as "Good functioning in all areas" before the onset of PNES, the most recent test after PNES onset showed that it had decreased to "Unable to function in almost all areas". We also collected information from her grandmother and teachers. As a result, we confirmed that she had definite difficulties in social communication that were not initially considered abnormal, abnormalities in sensory perception, as well as repetitive and restricted interests. We diagnosed her with ASD based on the Diagnostic and Statistical Manual of Mental Disorders, Fifth Edition (DSM-5) criteria. During her early childhood, she missed some developmental milestones, including joint attention, stranger anxiety, and pretend play. She also had restricted interests, including an obsession with calculators. However, her development was assessed as normal in medical checkups at the ages of 18 months and 3 years because she had no obvious delayed speech. Her intelligence and language abilities were normal (Wechsler Intelligence Scale for Children III: Verbal IQ =121, Performance IQ =111, Full Scale IQ =118). In contrast, her communication skills were precocious (particularly in lexicon) but pedantic, monotonous, and poor. She also displayed uncoordinated movements and clumsiness.

Furthermore, we identified several important recurring issues in the subject's history. After entering an elementary school at the age of 6 , she was often disturbed by slight sounds because of her auditory hypersensitivity. She also had difficulty in having interactive conversations and playing in groups. She was adaptable to a structured environment; for example, she felt comfortable in class where everything occurred on schedule and where students were required to follow a set of rules. She interpreted school rules literally and very strictly followed them. However, she had difficulty in understanding tacit agreements. She also had passive relationships with others, especially when there were no explicit rules, such as on weekends or during breaks between classes. She acted like a teacher at home and rebuked whoever broke the house rules (including her little brother). As her academic performance was very high in all subjects, except for physical 
education, her teachers viewed her as an excellent student. At an approximate age of 9, she started trying to adapt herself to social norms. She decided to be more social because she had no friends, unlike her classmates. She also struggled with fulfilling educational ideals, such as playing an active role in school. At the age of 10, she ran for class representative and actively tried to make friends. However, because of her intrusive attitude, these attempts failed and led to being teased by her classmates. A few months later, she showed signs of PNES as described earlier. We presume that stress from her social difficulties and repeated failures eventually resulted in her developing PNES.

The treatment approach that we employed focused on the patient's ASD traits. To avoid reinforcing the PNES symptoms, we advised the patient's parents and teachers not to give her undue attention. We discharged her in 2 weeks and continued her treatment in our outpatient clinic. We administered supportive psychotherapy to her and educated her parents and teachers about ASD and its supportive methods. Moreover, we asked them to let the subject wear earmuffs in school and at home to ameliorate her auditory hypersensitivity. She was also protected from bullying. Although she obsessively immersed herself in extracurricular matters, such as false accusation cases, we positively accepted her preoccupation as intellectual talent instead of considering it to be a restricted interest associated with ASD. Furthermore, we provided her with a place in which she was able to play with children in a small group despite her limited communication skills.

Although her PNES worsened temporarily when the subject attended school with her mother after being discharged, the number of seizures dramatically decreased within 2 weeks. Currently, she has focal seizures once or twice a year just after falling asleep, and the PNES have completely disappeared, without any signs for over a year. Her global functioning returned and has been maintained at pre-PNES levels, and she has begun to be accepted as an inarticulate but earnest scholar in school. Additionally, she has increased her self-affirmation, is able to better control her temper at home, and has become more adaptive in daily life.

\section{Discussion}

To the best of our knowledge, this is the first report of PNES in a child with ASD. Our case provides two important insights. First, PNES can result from psychological distress in children with undiagnosed ASD. Second, PNES in children might be a sign that they have undiagnosed ASD, and thus, being attentive to ASD symptoms in patients with PNES is crucial.
PNES can develop as a manifestation of psychological distress in children with undiagnosed ASD. Common psychiatric comorbidities for ASD in school-age children include anxiety disorders, mood disorders, obsessive-compulsive disorders, and disruptive behavioral disorders. ${ }^{14,15}$ However, in this case, ASD occurred with PNES. Using video-EEG and child psychiatric assessment, both the pediatric neurologists and the child psychiatrists collaborated to make a definitive diagnosis of PNES. The estimated prevalence of PNES in children who have suspected epilepsy varies from $1 \%$ to $9 \%$. However, these numbers are likely to be underestimated. ${ }^{9}$ Given that ASD and epilepsy co-occur in approximately $30 \%$ of individuals with ASD or epilepsy, ${ }^{11}$ a considerable number of ASD cases in children with PNES presumably exist, although no such reports have been published. Matsuo et $\mathrm{al}^{16}$ reported that ASD was undiagnosed in half of ASD children with epilepsy when their epilepsy was diagnosed. They also found that ASD had been overlooked for $>5$ years in onethird of these cases. Their findings support our contention that there are a large number of children with both ASD and PNES. Moreover, adult patients with PNES tend to talk less about their seizures and show less active struggles against seizure threats than patients with epilepsy. ${ }^{9,17}$ Although these tendencies are characteristics of adult PNES, they seem to resemble communication impairments that can be observed in both adults and children with ASD. For example, neither can effectively use facial expressions or gestures. Similarly, both adults and children with ASD answer poorly to open-ended questions because they have difficulty in understanding the intent of the questions. Their impaired communication skills can be hard to differentiate from the reticence of patients with PNES. The similarities between the two disorders may be a reason why diagnosing ASD in patients with PNES is extremely difficult and why overlooking ASD symptoms is common for clinicians when they examine PNES children with undiagnosed ASD. In our case, the subject had difficulty verbalizing her inner conflicts. She was viewed as an excellent student because she had learned coping strategies, such as being silent about her inner conflicts and strictly obeying rules. However, the distress caused by impaired communication and auditory hypersensitivity worsened with age as she was exposed to an increasingly complex social life. We presume that her psychological distress eventually became severe enough to trigger PNES. Therefore, we must seriously take into account criterion C for ASD in DSM-5, ${ }^{10}$ which mentions the possibility of ASD symptoms being concealed by learned strategies in later life. Moreover, when clinicians examine PNES children with undiagnosed ASD, they should 
be aware that distinguishing social communication deficits in ASD from the reticence exhibited by patients with PNES is difficult. Clinicians should also ask family members if the children have restricted patterns of interests or sensory hypersensitivity at home. Additionally, pediatric neurologists specializing in epilepsy are generally unfamiliar with ASD, while child psychiatrists are often inexperienced with epilepsy. This might be another factor that contributes to the lack of an ASD diagnosis in children with PNES. Therefore, both types of specialist must collaborate for more accurate diagnoses and assessments.

Our findings suggest that checking for signs of ASD in patients with PNES is important. Interventions for children with PNES are controversial, ${ }^{1,8}$ although one controlled study of the risk factors for pediatric PNES demonstrated that somatopsychiatric and adversity components can differentiate children with PNES from their siblings. ${ }^{18}$ However, no controlled studies on the treatment of childhood PNES have been reported. A recent controlled pilot clinical trial of adult PNES showed the effectiveness of eclectic psychotherapy. ${ }^{19}$ Additionally, several descriptive studies of childhood PNES have evaluated therapeutic approaches such as academic support for children and sensitive communication with their families, ${ }^{20}$ psychotherapy for children including confrontation, ${ }^{21}$ stress management techniques including relaxation therapy and cognitive approaches, ${ }^{8}$ and drug therapy for comorbid attention-deficit/hyperactivity disorder or separation anxiety. ${ }^{22}$

Because children with PNES manifest a variety of psychopathologies, conventional treatment for PNES must be tailored to each individual patient so that psychosocial stress can be reduced..$^{23}$ This conventional approach requires diverse expertise of people, including neurologists, psychologists, psychiatrists, psychotherapists, and social workers, who are not available at all clinical sites. However, in our case, we used a general and straightforward strategy that does not require such diverse resources, and PNES symptoms subsequently disappeared. We simply helped the parents and teachers to understand ASD characteristics and structured a nurturing environment for the child. School-age children with ASD often suffer from auditory hypersensitivity and poor social communication skills at school, and our treatment strategies targeted these problems. Because our therapeutic approach does not necessitate the varied expertise and resources required by the conventional treatment approach, it is much more economical and easier for therapists, parents, and teachers to implement.

\section{Conclusion}

To the best of our knowledge, this is the first report of PNES in a child with ASD, although we suspect that such cases are not so rare among those with PNES. PNES can occur in children with undiagnosed ASD and indeed might be related to the stress that results from such a situation. Consequently, clinicians should consider the possibility of undiagnosed comorbid ASD in patients with PNES, especially in those with epilepsy, and should be on the lookout for characteristic signs. Furthermore, they need to know that identifying impairments in social communication related to ASD is difficult in PNES children with undiagnosed ASD. They should also determine whether the children have restricted patterns of interests or sensory hypersensitivity at home. If signs of ASD are identified, the patient's environment should be structured, and the clinical staff should give sufficient attention to ASD characteristics, instead of exclusively focusing on bullying or academic and relationship problems.

Further studies are required to clarify the prevalence of ASD in the PNES population. The clinical course of PNES in our case suggests that treatment approaches focusing on ASD traits may have therapeutic potential in the treatment of PNES in children with ASD. A large controlled study is needed to fully evaluate the effectiveness of our clinical strategies.

\section{Acknowledgments}

The authors would like to thank the patient and her parents for their agreement to seek publication. Further thanks go to Dr Kuniko Ueda for her careful reading of the manuscript.

\section{Disclosure}

The authors report no conflicts of interests in this work.

\section{References}

1. Reilly C, Menlove L, Fenton V, et al. Psychogenic nonepileptic seizures in children: a review. Epilepsia. 2013;54(10):1715-1724.

2. Akagi H, House A. The clinical epidemiology of hysteria: vanishingly rare, or just vanishing? Psychol Med. 2002;32(2):191-194.

3. Wiseman H, Reuber M. New insights into psychogenic nonepileptic seizures 2011-2014. Seizure. 2015;29:69-80.

4. Kerr MP, Mensah S, Besag F, et al. International consensus clinical practice statements for the treatment of neuropsychiatric conditions associated with epilepsy. Epilepsia. 2011;52(11):2133-2138.

5. LaFrance WC Jr, Baker GA, Duncan R, et al. Minimum requirements for the diagnosis of psychogenic nonepileptic seizures: a staged approach A report from the International League Against Epilepsy Nonepileptic Seizures Task Force. Epilepsia. 2013;54(11):2005-2018.

6. Kim SH, Kim H, Lim BC, et al. Paroxysmal nonepileptic events in pediatric patients confirmed by long-term video-EEG monitoring - single tertiary center review of 143 patients. Epilepsy Behav. 2012;24(3): 336-340. 
7. Kanner AM. More controversies on the treatment of psychogenic pseudoseizures: an addendum. Epilepsy Behav. 2003;4(3):360-364.

8. Irwin K, Edwards M, Robinson R. Psychogenic non-epileptic seizures: management and prognosis. Arch Dis Child. 2000;82(6):474-478.

9. Reuber M. Psychogenic nonepileptic seizures: answers and questions. Epilepsy Behav. 2008;12(4):622-635.

10. Association AP. Diagnostic and Statistical Manual of Mental Disorders. 5th ed. Arlington, VA: American Psychiatric Publishing; 2013.

11. Mannion A, Leader G. Epilepsy in autism spectrum disorder. Res Autism Spectr Disord. 2014;8(4):354-361.

12. Ito H, Tani I, Yukihiro R, et al. Validation of an interview-based rating scale developed in Japan for pervasive developmental disorders. Res Autism Spectr Disord. 2012;6(4):1265-1272.

13. Takahashi K, Miyawaki D, Suzuki F, et al. Hyperactivity and comorbidity in Japanese children with attention-deficit/hyperactivity disorder. Psychiatry Clin Neurosci. 2007;61(3):255-262.

14. Gjevik E, Eldevik S, Fjaeran-Granum T, et al. Kiddie-SADS reveals high rates of DSM-IV disorders in children and adolescents with autism spectrum disorders. J Autism Dev Disord. 2011;41(6):761-769.

15. Kusaka H, Miyawaki D, Nakai Y, et al. Psychiatric comorbidity in children with high-functioning pervasive developmental disorder. Osaka City Med J. 2014;60(1):1-10.

16. Matsuo M, Maeda T, Sasaki K, et al. Frequent association of autism spectrum disorder in patients with childhood onset epilepsy. Brain Dev. 2010;32(9):759-763.
17. Schwabe M, Reuber M, Schondienst M, et al. Listening to people with seizures: how can linguistic analysis help in the differential diagnosis of seizure disorders? Commun Med. 2008;5(1):59-72.

18. Plioplys S, Doss J, Siddarth P, et al. A multisite controlled study of risk factors in pediatric psychogenic nonepileptic seizures. Epilepsia. 2014;55(11):1739-1747.

19. LaFrance WC, Baird GL, Barry JJ, et al. Multicenter pilot treatment trial for psychogenic nonepileptic seizures a randomized clinical trial. JAMA Psychiatry. 2014;71(9):997-1005.

20. Chinta SS, Malhi P, Singhi P, et al. Clinical and psychosocial characteristics of children with nonepileptic seizures. Ann Indian Acad Neurol. 2008;11(3):159-163.

21. Verrotti A, Agostinelli S, Mohn A, et al. Clinical features of psychogenic non-epileptic seizures in prepubertal and pubertal patients with idiopathic epilepsy. Neurol Sci. 2009;30(4):319-323.

22. Wyllie E, Glazer JP, Benbadis S, et al. Psychiatric features of children and adolescents with pseudoseizures. Arch Pediatr Adolesc Med. 1999; 153(3):244-248

23. LaFrance WC, Reuber M, Goldstein LH. Management of psychogenic nonepileptic seizures. Epilepsia. 2013;54:53-67.
Neuropsychiatric Disease and Treatment

\section{Publish your work in this journal}

Neuropsychiatric Disease and Treatment is an international, peerreviewed journal of clinical therapeutics and pharmacology focusing on concise rapid reporting of clinical or pre-clinical studies on a range of neuropsychiatric and neurological disorders. This journal is indexed on PubMed Central, the 'PsycINFO' database and CAS,

\section{Dovepress}

and is the official journal of The International Neuropsychiatric Association (INA). The manuscript management system is completely online and includes a very quick and fair peer-review system, which is all easy to use. Visit http://www.dovepress.com/testimonials.php to read real quotes from published authors. 